\title{
Comparative Study of Tadalafil and Tamsulosin as on therapy For Lower Urinary Tract Symptoms Due To Benign Hyperplasia of Prostate.
}

\author{
Dr Devendera Singh Pawar ${ }^{1}$, Dr Ashok Kumar ${ }^{2}$, Dr Santosh Kumar Singh ${ }^{3}$, \\ Dr Rajwardhan Singh: Mch Pursuing ${ }^{4}$, Dr Lokinder Yadav ${ }^{5}$, Dr Seema Mittal ${ }^{6}$ \\ ${ }^{1}$ Professor, Department Of Urology, Pt BD Sharma PGIMS, Rohtak. \\ ${ }^{2}$ Department Of Urology, Pt BD Sharma PGIMS, Rohtak \\ ${ }^{3}$ Professor, Department Of Urology, Pt BD Sharma PGIMS, Rohtak \\ ${ }^{4}$ Department Of Urology, Pt BD Sharma PGIMS, Rohtak \\ ${ }^{5}$ Department Of Urology, Pt BD Sharma PGIMS, Rohtak \\ ${ }^{6}$ Assistant Professor (Microbiology), BPS Khanpur Medical College(W), Sonepat
}

Objectives: The U.S. Food and Drug Administration approved tadalafil in 2011 to treat the signs and symptoms of Lower Urinary Tract symptoms (LUTS) due to benign prostatic hyperplasia (BPH). Tadalafil is a phosphodiesterase-5 (PDE5) inhibitor improves erectile function by increasing the amount of cyclic guanosine monophosphate in the smooth muscle of the corpus cavernosa. This study aims for the comparison of tadalfil with other established drug tamsulosin in south asian population

Methods: A total of 120 patient presenting with lower urinary tract symptoms( LUTS) due to BPH were selected and randomized with card method to receive either $5 \mathrm{mg}$ tadalafil daily or $0.4 \mathrm{mg}$ tamsulosin daily. Base line $Q$ max, PVR(post void residual), IPSS(International prostate symptom score), IIEF(International index of erectile function-erectile function) were noted at start of study and at end of 12 weeks. Patient with history of drug treatment, history of prostate surgery and raised PSA were excluded from study.

Results: Both group were comparable. Both tamsulosin and tadalafil improved symptoms of LUTS. But tamsulosin is slightly more effective than tadalafil but not significantly $(p>0.05)$ in relieving LUTS. But only tadalafil significantly improved IIEF $(p<0.05)$. Both treatments were tolerated well. There was component of subclinical erectile dysfunction in 36 out of 60.

Conclusion: Symptoms of erectile dysfunction and LUTS frequently occurs together. These could well be treated with monotherapy of tadalafil. It is still not clear significance of treating subclinical erectile dysfunction.

\section{Introduction}

$\mathrm{BPH}$ is a chronic progressive non-malignant disease in men associated with LUTS.${ }^{1}$ As the prostate increases in size with age, it may occlude the lumen of the prostatic urethera, causing mechanical urinary tract obstruction. $^{2}$

Two kind of bladder outlet obstruction had been described in literature one is static component which is due to prostate enlargement and can be manage by surgery and to some extent by antiandrogen drugs. Another is dynamic component which is modifiable. This component is managed by drugs in form of alpha-1 blockers and phosphodiestrase inhibitors. As a result, $\alpha 1-\mathrm{AR}$ antagonists are widely recognized as the first-line pharmacotherapy for BPH treatment. ${ }^{3}$

Alpha blockers like prazosin, alfuzosin, tamsulosin and silodosin had been approved for use in patients of LUTS due to BPH and had been found to improve symptoms of LUTS. However Tadalafil is most recent addition this armantarium and only recently been approved by FDA for use in LUTS due to BPH. Mechanism of action of tadalafil is that it inhibit PDE 5 inhibtion and improve erectile function by increasing the amount of cyclic guanosine monophosphate in the smooth muscle of the corpus cavernosa. This action increases penile blood flow, resulting in penile erection during sexual stimulation. PDE5 inhibition also affects concentration of cyclic guanosine monophosphate in the smooth muscle of the prostate, the bladder, and their vascular supply, but the precise mechanism for reducing BPH symptoms has not been determined. ${ }^{4}$

Aging is a key risk factor for the development of male ED. Kinsey showed that the prevalence of ED increased with age from $0.1 \%$ at $20 \mathrm{yr}$ to $75 \%$ at $80 \mathrm{yr}^{5}$ A half century later, the MMAS (12) largest known survey on sexual dysfunction showed that the prevalence of ED increased from 39\% in men in their 40s to 67\% for men in their 70s. Using the same questionnaire as the MMAS study, the Cross-National Epidemiological Study was conducted in four different countries with varying cultures: Brazil, Italy, Japan, and Malaysia ${ }^{6}$. The results confirmed the findings of the MMAS in these different countries. 
Prevalence of erectile dysfunction and LUTS due to BPH increases with old age. Tadalafil is marketed to improve both these symptoms. This drug has been used for erectile dysfunction but effect on LUTS is found in post marketing survellience. Now we want to compare effectiveness of tadalafil with a established drug tamsulosin for the indication of LUTS due to BPH.

\section{Methods}

This study is conducted by department of urology in 2015 after obtaining clearance from local ethics committee of university and did not involve any unethical practise. This study is randomized controlled prospective single blind study .

After obtaining written informed consent, patients were registered and divided into the following two groups randomally by card method: Tamsulosin group (group T), receiving $0.4 \mathrm{mg}$ of tamsulosin hydrochloride once daily in the evening, and tadalafil group (group D), receiving $5 \mathrm{mg}$ of tadalafil in the evening after meal. A total of 120 consenting male patients with LUTS with BPH who visited outpatient department of Surgery and Urology and satisfied the followings condition: Men age more than 45 years, storage symptoms (increased day time frequency, urgency and nocturia and or voiding symptoms (hesitancy, incomplete voiding, impaired stream or interruption of stream), nocturia $>2$, maximum flow rate $<15 \mathrm{ml} / \mathrm{sec}$ with a voided volume of at least $150 \mathrm{ml}$, postvoid residual urine less than $100 \mathrm{ml}$ by transabdominal ultrasound, IPSS $>13$ points, international prostatic symptom bother score $>3$ points were enrolled in this study. Patients who had previous prostate surgery, severe visceral disease, postural hypotension, neurogenic bladder dysfunction, suspected prostate cancer, urethral stricture disease, history of pelvic irradiation, bladder neck disease, acute bacterial prostatitis, acute urinary tract infection, urolithiasis, concomitant medication that may alter the voiding pattern before inclusion (calcium antagonists like monoamineoxidase inhibitors or anticholinergic drugs), active hematuria, renal insufficiency (serum creatinine $>2.0 \mathrm{mg} / \mathrm{dl}$ ), severe hepatic impairment (transaminases $>2$ times the upper normal limit and total bilirubin $>1.5 \mathrm{mg} / \mathrm{dl}$ ), patients on antipsychotic medications, insulin dependant diabetes mellitus, history of severe heart disease were excluded. Evaluation included clinical determination of IPPS, QoL, IIEF, maximum flow rate $(\mathrm{ml} / \mathrm{s})$, time to maximum flow, average flow rate, average flow time by uroflometry performed on Laborie Urocap III uroflowmeter with Bluetooth technology, postvoid residual urine volume and prostate size by ultrasonographically at inclusion, 1 month and 3 month respectively.

Students $t$ test is used for statistical analyses.

\section{Results}

A total of 120 patients were included in the study. They were compared in terms of age, prostate size, postvoid residual urine, uroflow parameters IPSS and IIEF score.

Mean age of patients was comparable $68.4+/-12.3$ for tamsulosin group and 67.2+/-11.8 in tadalafil group. Mean prostate size was 56 and 52 respectively. Datas for Prostate size, postvoid residue uroflow parameters and IPSS scores are shown in Tables 1,2,3 and 4.

Both drugs does not decrease prostate size however tamsulosin improve post void residual while tadalafil did not. Both drugs do improve LUTS symptoms and uroflow parameters but only tadalafil improve erectile dysfunction.

Out of 120 patients only 86 patients were sexually active. Out of 86 , fourty two were in tamsulosin group and 44 were in tadalafil group. Both drugs were well tolerated no patient were needed to stop drug due to side effects. Both the drugs well tolerated. In tadalfil group except for 2 patient complained of mild headache, one of patients had nasal cogestion and one other body pain. None of patients stopped drugs. No major side effect was reported by patient on tamsulosin group except for two patient reporting weakness and one had mild headache.

\section{Discussion}

LUTS and Erectile dysfunction are highly related diseases as both had increased incidence and prevalence with increasing age. ${ }^{7,8}$ For a long time both were considered as two different diseases and were treated separately. Many times erectile dysfunction part is neglected both by patient and physician as it is considered normal part of ageing and untreatable. Treatment used for LUTS also has negative impact on sexual function of male patient and worsen the disease.

The Multinational Survey of the Ageing Male (MSAM7), one of the largest population based studies of ageing men conducted, evaluated the associations between age, LUTS, concomitant comorbidities and male sexual dysfunction in > 12,000 men in the United States and Europe. In MSAM7, the overall prevalence of LUTS was 90\%, while the overall prevalence of ED (erectile dysfunction) and EjD (ejaculatory dysfunction) was $49 \%$ and $46 \%$, respectively. The rate of both ED and EjD was significantly dependent on age and correlated highly with the severity of LUTS . 
PDE 5 inhibitors are the well known treatment modality for the symptoms of ED and used as first line of management. FDA has approved tadalafil $(5 \mathrm{mg})$ for LUTS with the objective to treat both the diseases simulataneouly and without worsening one of diseases. ${ }^{10-12}$

PDE5 inhibitors acts by upregulation of the NO/cGMP activity. Preclinical studies reported partial reversal of nor-epinephrine- and endothelin-1-reduced prostatic tissue contraction ${ }^{13}$ and an anti-proliferative effect on cultured prostate and bladder smooth muscle cells ${ }^{14}$. These mechanisms may decrease smooth muscle tension in the prostatic stroma and capsule and attenuate cellular proliferation associated with prostate/bladder hypertrophy respectively. Tadalafil had shown improved blood flow to prostate on color doppler ultrasound ${ }^{15}$ There are many established drugs for LUTS due to BPH. Alpha blockers and Dutasteride/ finasteride are most popular among these.

Selective $\alpha_{1}$-blockers are most polular first line treatment. It is considered the standard of care ${ }^{16}$ and the most effective medical therapy for LUTS due to benign prostate enlargement.. Their use is based on the presence of contractile tissue in the prostate mediated via $\alpha_{1}$-adrenergic receptors abundant in the bladder neck, prostate capsule, and stroma. This dynamic component demonstrated to contribute approximately $40 \%$ of outflow obstruction. ${ }^{17,18}$ impotence. $^{19}$

In an open-label tamsulosin study, $30 \%$ of subjects reported abnormal ejaculation and $6 \%$ reported

McVary and Roehrborn on the double-blind, placebo-controlled study and dose finding study respectively reported no change in PVR( post voidal residual volume) in the tadalafil group ${ }^{20-1}$ Our study also demonstrated no change in PVR even at end of 3 months.

Porst and colleagues reported improvement in IPSS but changes in peak urinary flow (Qmax) and PVR were small and not clinically meaningful ${ }^{22}$. In this context, Brock and colleagues reported on another paper based on the same database of patients that changes in BPH-LUTS after 12 weeks of treatment with placebo or various doses of once-daily tadalafil were similar in men with or without comorbid ED. After 12 weeks, changes in IPSS in men with ED and without ED were not significantly different (subgroup/interaction $\mathrm{p}$ values: $0.352 / 0.644) .{ }^{23}$.It shows that effect on LUTS is independant of the effect on ED. Our study also shows similar findings.

All of these studies reported that tadalafil is not only efficacious but also a safe treatment. As in the classic ED trials, the most common side effects were headache, dyspepsia, nasal congestion, flushing and back pain. ${ }^{24}$.

Recently, data on the combination of tadalafil with finasteride (a 5a-reductase inhibitor) have been published ${ }^{25}$. IPSS improvement is significantly higher in the combination group compared with the finasterideonly group at the . As expected, IIEF improved significantly in the combination group $(\mathrm{p}<0.001)$. Combination therapy was well tolerated and most adverse events were mild/moderate.

ED and BPH-associated LUTS are epidemiologically linked and share common pathophysiological pathways. . . Tadalafil is only approved drug available today which can be used for both symptoms. However whether to treat or not subclinical erectile dysfunction or not is debatable. However If sexual function one of biggest priority of patient more so in young patient, patients with sexual dysfunction side effects and with mild to moderate LUTS Tadalafil can be drug of choice in such situation. One can add other drugs to it if need arises.

However further studies are required for the study of combination of other drugs like alfuzosin with tadalafil.

\section{References}

[1]. Chapple C. Medical treatment for benign prostatic hyperplasia. BMJ 1992; 304: 1198-9.

[2]. Clifford GM, Farmer RDT. Medical therapy for benign prostatic hyperplasia: a review of the literature. Eur Urol 200 AUA Practice Guidelines Committee.

[3]. AUA guideline on management of benign prostatic hyperplasia. Chapter 1: Diagnosis and treatment recommendation. J Urol 2003; 170: 530-47.0; 38:2-19.

[4]. Andersson KE, Lepor H, Wyllie MG. Prostatic alpha 1-adrenoceptors and uroselectivity. Prostate 1997; 30: 202-15.

[5]. . Kinsey AC, Pomeroy WB, Martin CE. Sexual behavior in the human male. WB Sanders, Philadelphia, 1948.

[6]. McKinlay JB, DiGruttolo L, Glasser D, et al. International differences in the epidemiology of male erectile dysfunction. Int J Impotence Res 1998; 10(Suppl 3): S42.

[7]. Robertson C., Link C., Onel E., Mazzetta C., Keech M., Hobbs R., et al. (2007) The impact of lower urinary tract symptoms and comorbidities on quality of life: the BACH and UREPIK studies. BJU Int 99: 347-354

[8]. Gacci M., Eardley I., Giuliano F., Hatzichristou D., Kaplan S., Maggi M., et al. (2011) Critical analysis of the relationship between sexual dysfunctions and lower urinary tract symptoms due to benign prostatic hyperplasia. Eur Urol 60: 809-825. Rosen R., Altwein J., Boyle P., Kirby R., Lukacs B., Meuleman E., et al. (2003) Lower urinary tract symptoms and male sexual dysfunction: the Multinational Survey of the Aging Male (MSAM-7). Eur Urol 44: 637-649.

[9]. Hatzimouratidis K., Amar E., Eardley I., Giuliano F., Hatzichristou D., Montorsi F., et al. (2010) Guidelines on male sexual dysfunction: erectile dysfunction and premature ejaculation. Eur Urol 57: 804-814. 
[10]. McVary K., Monnig W., Camps J., Jr, Young J., Tseng L., Van Den Ende G. (2007a) Sildenafil citrate improves erectile function and urinary symptoms in men with erectile dysfunction and lower urinary tract symptoms associated with benign prostatic hyperplasia: a randomized, double-blind trial. J Urol 177: 1071-1077.

[11]. McVary K., Roehrborn C., Kaminetsky J., Auerbach S., Wachs B., Young J., et al. (2007b) Tadalafil relieves lower urinary tract symptoms secondary to benign prostatic hyperplasia. J Urol 177: 1401-1407.

[12]. Kedia G., Uckert S., Kedia M., Kuczyk M. (2009) Effects of phosphodiesterase inhibitors on contraction induced by endothelin-1 of isolated human prostatic tissue. Urology 73: 1397-1401.

[13]. Morelli A., Sarchielli E., Comeglio P., Filippi S., Mancina R., Gacci M., et al. (2011) Phosphodiesterase type 5 expression in human and rat lower urinary tract tissues and the effect of tadalafil on prostate gland oxygenation in spontaneously hypertensive rats. J Sex Med 8: 2746-2760.

[14]. Bertolotto M., Trincia E., Zappetti R., Bernich R., Savoca G., Cova M. (2009) Effect of tadalafil on prostate haemodynamics: preliminary evaluation with contrast-enhanced US. Radiol Med 114: 1106-1114

[15]. Kirby RS. The natural history of benign prostatic hyperplasia: what have we learned in the last decade?Urology. 2000;56(5) suppl $1: 3-6$.

[16]. Roehrborn CG. Introduction. Urology. 2000;56(suppl 5A):1-2.

[17]. Höfner K, Claes H, De Reijke TM, et al. Tamsulosin $0.4 \mathrm{mg}$ once daily: effect on sexual function in patients with lower urinary tract symptoms suggestive of benign prostatic obstruction. Eur Urol.1999;36:335-341.

[18]. Narayan P, Lepor H. Long-term, open-label, phase III multicenter study of tamsulosin in benign prostatic hyperplasia. Urology. 2001;57:466-470.

[19]. Debruyne FMJ. Alpha blockers: are all created equal? Urology. 2000;56(suppl 5A):20-22.

[20]. Roehrborn C., McVary K., Elion-Mboussa A., Viktrup L. (2008) Tadalafil administered once daily for lower urinary tract symptoms secondary to benign prostatic hyperplasia: a dose finding study. J Urol 180: 1228-1234.

[21]. Porst H., McVary K., Montorsi F., Sutherland P., Elion-Mboussa A., Wolka A., et al. (2009) Effects of once-daily tadalafil on erectile function in men with erectile dysfunction and signs and symptoms of benign prostatic hyperplasia. Eur Urol 56: 727-735.

[22]. Brock G., Broderick G., Roehrborn C., Xu L., Wong D., Viktrup L. (2013) Tadalafil once daily in the treatment of lower urinary tract symptoms (LUTS) suggestive of benign prostatic hyperplasia (BPH) in men without erectile dysfunction. BJU Int, in press.

[23]. Porst H., Roehrborn C., Secrest R., Esler A., Viktrup L. (2013b) Effects of tadalafil on lower urinary tract symptoms secondary to benign prostatic hyperplasia and on erectile dysfunction in sexually active men with both conditions: analyses of pooled data from four randomized, placebo-controlled tadalafil clinical studies. J Sex Med 10: 2044-2052.

[24]. Donatucci C., Brock G., Goldfischer E., Pommerville P., Elion-Mboussa A., Kissel J., et al. (2011) Tadalafil administered once daily for lower urinary tract symptoms secondary to benign prostatic hyperplasia: a 1-year, open-label extension study. BJU Int 107: $1110-1116$.

[25]. Casabe A., Roehrborn C., Da Pozzo L., Zepeda S., Henderson R., Sorsaburu S., et al. (2014) Efficacy and safety of the coadministration of tadalafil once daily with finasteride for 6 months in men with lower urinary tract symptoms and prostatic enlargement secondary to benign prostatic hyperplasia. J Urol 191: 727-733.

Tables

\begin{tabular}{|l|l|l|l|l|}
\hline & Baseline & 1 month & 3 month & p value \\
\hline Group1:Tamsulosin & $56.00 \pm 6.29$ & $54.00 \pm 6.47$ & $54.00 \pm 7.16$ & 0.15 \\
\hline Group 2: Tadalafil & $52.00 \pm 7.18$ & $52.00 \pm 7.01$ & $52.00 \pm 7.17$ & 0.17 \\
\hline
\end{tabular}

Table 1 Prostate size

\begin{tabular}{|c|l|l|c|l|}
\hline & Baseline & 1 month & 3 month & p value \\
\hline Group 1: Tamsolusin & $86.00 \pm 11.20$ & $30.00 \pm 10.97$ & $10.00 \pm 6.55$ & $\leq 0.001$ \\
\hline Group 2: Tadalafil & $80.0 \pm 15.06$ & $73.00 \pm 10.74$ & $67 \pm 6.87$ & 0.065 \\
\hline
\end{tabular}

Table 2 Post void residual volumes

\begin{tabular}{|c|c|c|c|c|c|c|c|c|c|c|c|c|c|c|c|}
\hline \multicolumn{9}{|c|}{ Tamsulosin } & \multicolumn{7}{|c|}{ Tadalafil } \\
\hline & & \multicolumn{2}{|c|}{ Inclusion } & \multicolumn{2}{|c|}{ Month 1} & \multicolumn{2}{|c|}{ Month 3} & \multirow{2}{*}{\multicolumn{2}{|c|}{$\begin{array}{l}\text { p } \\
\text { value } \\
\leq 0.001\end{array}$}} & \multicolumn{2}{|c|}{ Inclusion } & \multicolumn{2}{|c|}{ Month 1} & Month 3 & $\begin{array}{l}\text { p } \\
\text { value }\end{array}$ \\
\hline \multicolumn{2}{|c|}{$\begin{array}{l}\text { Maximum flow rate } \\
(\mathrm{ml} / \mathrm{s})\end{array}$} & \multicolumn{2}{|c|}{$9.4 \pm 0.82$} & \multicolumn{2}{|c|}{\begin{tabular}{ll|}
12.06 & \pm \\
1.35 & \\
\end{tabular}} & \multicolumn{2}{|c|}{\begin{tabular}{ll|}
12.23 & \pm \\
1.22 & \\
\end{tabular}} & & & \multicolumn{2}{|c|}{$9.2 \pm 1.47$} & \multicolumn{2}{|c|}{$\begin{array}{ll}10.8 & \pm \\
2.13 & \end{array}$} & $\begin{array}{l}10.5 \pm \\
1.79 \\
\end{array}$ & 0.08 \\
\hline \multicolumn{2}{|c|}{$\begin{array}{l}\text { Average flow rate } \\
(\mathrm{ml} / \mathrm{s})\end{array}$} & \multicolumn{2}{|c|}{$5.4 \pm 0.77$} & \multicolumn{2}{|c|}{$6.8 \pm 0.64$} & \multicolumn{2}{|c|}{$6.9 \pm 0.61$} & \multicolumn{2}{|c|}{$\leq 0.001$} & \multicolumn{2}{|c|}{$5.2 \pm 0.79$} & \multicolumn{2}{|c|}{$5.3 \pm 0.66$} & $5.4 \pm 0.74$ & 0.06 \\
\hline \multicolumn{2}{|c|}{$\begin{array}{l}\text { Time to Maximum } \\
\text { flow }\end{array}$} & \multicolumn{2}{|c|}{$\begin{array}{ll}38.0 & \pm \\
4.87 & \\
\end{array}$} & \multicolumn{2}{|c|}{$\begin{array}{|ll|}32.1 & \pm \\
3.35 & \\
\end{array}$} & \multicolumn{2}{|c|}{$30.2 \quad \pm$} & \multicolumn{2}{|c|}{$\leq 0.001$} & \multicolumn{2}{|c|}{$\begin{array}{ll}47.35 & \pm \\
6.57 & \\
\end{array}$} & \multicolumn{2}{|l|}{$\begin{array}{l}35.7 \\
\pm 4.47 \\
\end{array}$} & $\begin{array}{ll}33.60 \quad \pm \\
4.39\end{array}$ & 0.045 \\
\hline \multicolumn{2}{|c|}{ Average flow time } & \multicolumn{2}{|c|}{\begin{tabular}{ll|}
12.0 & \pm \\
2.89 & \\
\end{tabular}} & \multicolumn{2}{|c|}{$8.4 \pm 2.00$} & \multicolumn{2}{|c|}{$\begin{array}{l}.65 \\
\pm 1.79 \\
\end{array}$} & \multicolumn{2}{|c|}{$\leq 0.001$} & \multicolumn{2}{|c|}{$12.4 \pm 3.26$} & \multicolumn{2}{|c|}{$\begin{array}{ll}11.34 \quad \pm \\
2.03\end{array}$} & $\begin{array}{l}10.2 \\
2.10 \\
\end{array}$ & $\mathbf{0 . 0 3 8}$ \\
\hline Scores & \multicolumn{2}{|l|}{ Inclusion } & Month & & Month & & p valu & & & usion & & th 1 & & onth 3 & p value \\
\hline IPSS & $20.5 \pm 3.3$ & & $14.0 \pm 2$ & & $\begin{array}{l}12.00 \\
1.94 \\
\end{array}$ & & $\leq 0.00$ & & & \pm 2.79 & & \pm 1.9 & & $.0 \pm 1.25$ & $\leq 0.001$ \\
\hline IIEF-5 & $11.4 \pm 0.4$ & & $12.0 \pm 0$ & & $12.2 \pm$ & .48 & 0.525 & & & \pm 0.62 & & \pm 0.58 & & $.2 \pm 0.48$ & $\leq 0.001$ \\
\hline
\end{tabular}

Original Table : IPSS and IIEF Scores of both groups. 